\title{
Cervical sampling for diagnosis of genital chlamydial infection with a new brush device
}

\author{
L-O Svensson, M Domeika, P-A Mårdh
}

\begin{abstract}
Objectives-to compare a new sampling device, a brush, Accellone-Multi-Instrument (AMI), with a dacron-tipped swab for detection of Chlamydia trachomatis in endocervical specimens, and to evaluate if consecutive multiple cervical sampling as compared with such a single specimen would increase the sensitivity. Methods-501 females attending an STD clinic and 172 females attending a family planning clinic were examined prospectively. Two cervical specimens were collected from each woman. $C$ trachomatis were detected by culture or enzyme immunoassay (IDEIA-III). Positive EIA samples were confirmed by a direct immunofluorescent test.

Results-When cervical specimens were collected with the brush as the first device, $92 \%$ of the culture-positive cases were detected, and when the samples were collected with the dacron-tippedswab first, $84 \%$ of the culture-positive cases were detected $(p<0.05)$. The first collected specimen detected $89 \%$ of the culture-positive cases and $81 \%$ of those that were positive by IDEIA.

Conclusions-The study indicates that the AMI brush is superior to non-toxic, dacron-tipped swabs for detection of $C$ trachomatis in cervical samples by cell culture but not by ELISA, and that the sensitivity could be improved by analysing multiple cervical samples.
\end{abstract}

(Genitourin Med 1993;69:397-399)

Institute of Obstetrics \& Gynecology,

University Hospital,

Uppsala, Sweden and Department of

Obstetrics \&

Gynecology, Central

Hospital, Västerás, Sweden

L-O Svensson

Institute of Clinical

Bacteriology,

University of Uppsala, Uppsala, Sweden

M Domeika

P-A Mårdh

Address correspondence to: Lars-Olof Svensson,

Kvinnokliniken

Centralsjukhuset S-721 89,

Västerås, Sweden

Accepted for publication 8 August 1993

\section{Introduction}

The means to detect genital infection by $C$ trachomatis is still not optimal. Thus it has been estimated that only approximately $70-80 \%$ of infected cases will be detected by presently available diagnostic techniques. ${ }^{1-2}$

A number of factors can influence the liklihood of detecting the organism in clinical samples, such as the type of specimen analysed (cervical, urethral, voided urine etc), the sampling device and sampling technique used, the storage, and transport conditions as well as the laboratory analytical technique used. ${ }^{13}$

The purpose of this study was to compare a new synthetic sampling brush, the Accellone-Multi-Instrument, with a dacrontipped swab. Furthermore, to evaluate if consecutive multiple cervical sampling as compared with such a single specimen would increase the detection rate for $C$ trachomatis from the cervix when analysed either with cell culture or ELISA.

\section{Material and methods}

Study populations

Specimens were collected from 501 females attending a sexually transmitted disease clinic (Group A), who were aged 18-35 years (mean 25.0), many of whom had symptoms. Also included in the study were 172 asymptomatic females (Group B) attending a family planning clinic for contraceptive advice, between the ages of 16-35 years (mean age $23 \cdot 4$ years).

\section{Specimen collection}

Two cervical specimens were collected from each woman, one specimen with a new type of brush, the Accellone-Multi-Instrument ${ }^{\circledR}$ (AMI) (Medscand, Malmö, Sweden) and the another specimen with a dacron-tipped swab. The former is a brush with a thin plastic shaft with its tip covered with soft bristles.

Before taking the samples the exocervix was cleansed with a dry cotton pad. On even calender dates the dacron-tipped swab was used first and vice versa on odd calender dates.

The specimens were placed in the transport medium supplied by the kit procedure (Novo Bio Labs) of the enzyme immunaoassay used or in a sucrose-phosphate (2-SP) buffer for tissue culture studies.

\section{Detection of chlamydia trachomatis}

Within 72 hours of sampling, samples from Group A were inoculated into cycloheximidetreated McCoy cell cultures.

Samples from Group B were analysed in an enzyme immunoassay, (IDEIA-III (Novo Bio Labs)). Positive samples were confirmed by a direct immunofluorescense test (DIF), (MicroTrak (Syva). Only samples positive either by culture or in both the IDEIA and the DIF tests were regarded as true positives.

\section{Results}

Of the 673 women included in the study, $85(12.6 \%)$ were found to be infected by $C$ trachomatis. Of the 501 females in Group A, $64(12 \cdot 8 \%)$ were positive as evidenced by culture. Of the 501 samples collected by use of a dacron-tipped swab, $51(10 \cdot 2 \%)$ were culture-positive. They constituted $79.7 \%$ 
Table 1 Comparison of a brush sampling device (Accelone-Multi-Instrument) and a dacron-tipped swab for detection of Chlamydia trachomatis by culture in group $A(N=501)$.

\begin{tabular}{lllll}
\hline Sampling device & \multicolumn{4}{l}{ Culture test } \\
\hline Brush & + & + & - & - \\
Swab & + & - & + & - \\
Total & 46 & 13 & 5 & 437 \\
\hline
\end{tabular}

In total, 59 and 51 of the chlamydia positive specimens had been sampled by the brush and the dacron-tipped swab, respectively.

of the cases detected to be infected with $C$ trachomatis.

With the AMI brush, 59 (11.8\%) were culture-positive, i.e. $92 \cdot 2 \%$ of all chlamydia cases detected in Group A (table 1).

In Group B, $21(12 \cdot 2 \%)$ of the 172 women were chlamydia-positive in IDEIA-111 in all cases confirmed by DIF. Both the dacrontipped swab and the AMI brush revealed 17 $(9.9 \%)$ of the chlamydia-infected cases which were $81 \%$ of those found to be infected in Group B (table 2).

When samples were taken by the dacrontipped swab before the brush in Group A, 21 of $25(84 \%)$ chlamydia-positive cases were detected by the first specimens, while when the AMI brush was the first device used, 36 of $39(92 \%)$ specimens were culturepositive $(\mathrm{p}<0.05)$ (table 3$)$.

In Group B, 11 specimens were ELISApositive when the dacron-tipped swab was the first device used, while this was true for 10 specimens when the AMI brush was used first (table 4).

The first collected specimens detected $86 \%$ $(57 / 66)$ of the culture-positive cases and $81 \%(17 / 21)$ of those who were positive by IDEIA-111.

No samples collected with the AMI brush or the dacron-tipped swab were found to be cytotoxic.

Traces of blood were observed in 297 (44\%) of the samples collected by the AMI device and in $152(23 \%)$ of those collected by the dacron-tipped swab.

\section{Discussion}

The detection of the intracellular organism $C$ trachomatis in tissue cell culture is related to the quantity of epithelial cells in the specimen collected. ${ }^{1}$

The detection rate is dependent on the amount of chlamydia organisms (or antigen) present in the specimen and the toxicity of

Table 2 Comparison of a brush sampling device (Accellone-Multi-Instrument) and a dacron-tipped swab used for detection of Chlamydia trachomatis by immunofluorescent-confirmed enzyme immunoassay $(I D E L A-111)(N=172)$.

\begin{tabular}{lclll}
\hline \multicolumn{4}{l}{ Sampling device } & \multicolumn{4}{l}{ Immunoassay } \\
\hline Brush & + & + & - & - \\
Swab & + & - & + & - \\
Total & 13 & 4 & 4 & 151 \\
\hline
\end{tabular}

In total, 17 chlamydia-positive specimens were detected in samples collected both by the brush and the dacron-tipped swab.
Table 3 Comparison of detection rate of Chlamydia trachomatis by culture between first and second consecutive sampling occasion in women sampled by a brush device (Accellone-Multi-Instrument) and a dacron-tipped swab. $(N=501)$

\begin{tabular}{llllll}
\hline $\begin{array}{l}\text { Sampling } \\
\text { device }\end{array}$ & $\begin{array}{l}\text { Sampling } \\
\text { order }\end{array}$ & Result & & & \\
\hline Brush & 1 & + & + & - & - \\
Swab & 2 & + & - & + & - \\
Total & & 27 & 9 & 3 & 209 \\
Swab & 1 & + & + & - & - \\
Brush & 2 & + & - & + & - \\
Total & & 19 & 2 & 4 & 228 \\
\hline
\end{tabular}

In total, 36 women were chlamydia-positive in the first sampling from the cervix when the sampling was made with the pling from the cervix when the sampling was made with the
brush, while 21 women were positive when the dacron-tipped swab was first to be used $(\mathrm{p}<0.05)$.

the sampling device is a factor which influences the outcome of culture studies. ${ }^{3}$ The recovery rate has been reported to be improved by using a brush device, the Cytobrush $^{\otimes}$ compared with dacron- or cottontipped swabs. ${ }^{4-7}$ The brushes yield an increased number of epithelial cells as compared with ordinary swabs in samples collected from the cervical channel. ${ }^{8}$ The same is true for the number of elementary bodies (EB) of $C$ trachomatis detected in such samples analysed by DIF. However, the advantage with the brush device has not been confirmed in other studies. ${ }^{8-13}$

The brush can induce bleeding from the friable cervical channel ${ }^{11}$ and a cytotoxic effect on McCoy cells, when samples have been contaminated with blood, has been reported. Bleeding occurred in $44 \%$ of the the sampled cases using the AMI-brush and in $23 \%$ of the cases using the dacron-tipped swab. The bleeding did not seem to affect the detection of $C$ trachomatis

However, samples taken with the AMIbrush has a higher sensitivity for detection of $C$ trachomatis by tissue cell cultures than when dacron-tipped swabs were used, which may be explained by the former sampling result in a higher number of epithelial cells in the specimen.

Dunlop et $a l^{14}$ found that multiple endocervical swabs increased the sensitivity of detecting genital infection by $C$ trachomatis. They found that the first collected swab iden-

Table 4 Comparison of the detection rate of Chlamydia trachomatis between first and second consecutive sampling occasion from the cervix in specimens collected by a brush device (Accellone-Multi-Instrument ${ }^{\circledR}$ ) and a dacrontipped swab when analysed by IDELA-111 and confirmed by direct immunofluorescens (Mikro Trak) $(N=172)$.

\begin{tabular}{llllll}
\hline $\begin{array}{l}\text { Sampling } \\
\text { device }\end{array}$ & $\begin{array}{l}\text { Sampling } \\
\text { order }\end{array}$ & Result & & & \\
\hline Brush & 1 & + & + & - & - \\
Swab & 2 & + & - & + & - \\
Total & & 6 & 2 & 2 & 77 \\
Swab & 1 & + & + & - & - \\
Brush & 2 & + & - & + & - \\
Total & & 7 & 2 & 2 & 74
\end{tabular}

In total, 8 women were chlamydia-positive when the first sam pling from the cervix was made with the brush, while 9 women were positive when the dacron-tipped swab was firs to be used. (Not significant). 
tified $73 \%$, the second an additional $17 \%$, and the third swab still $10 \%$ more culturepositive women. They concluded that in culture studies, samples obtained by use of a single swab underestimate the prevalence of genital chlamydial infection. In other studies $^{1015}$, only a marginal difference by multiple and single sampling has been demonstrated.

In the present study only $89 \%$ of the chlamydia-infected women in group $\mathrm{A}$ and only $81 \%$ of those in group B were detected with aid of the first collected specimen. That is, about $10-20 \%$ of the infected cases, would have been missed by culture and twice as many would not have been detected in ELISA if only a single sample had been obtained.

Sampling from the urethra in addition to the cervix in asymptomatic women, add less than $10 \%$ of chlamydia-positive cases as compared with when only cervical sampling is made. ${ }^{1416}$ Urethral sampling is, however, often painful and may cause urethritis symptoms because of injury of the urethral mucosa. Two consecutive cervical swabs would be effective in to diagnosing genital chlamydial infection without being painful. Thus, two consecutive samples added 11 $(15 \%)$ positive cases as compared with when only one cervical sample (collected by the aid of the dacron-tipped swab or the AMI device) was analysed.

There was no difference in the subjective feeling of discomfort after sampling with the AMI and the dacron-tipped swab.

To sum up, our study demonstrated that the Accellone-Multi-Instrument is equal or superior to non-toxic dacron-tipped swabs for detection of $C$. trachomatis by cell culture of endocervical specimens and that duplicate endocervical specimens increases the sensitivity both when analysing the samples by culture and EIA in cervical samples.
1 Kellogg JA. Clinical and laboratory considerations of culture vs antigen assays for detection of Chlamydia trachomatis from gen

2 Taylor-Robinson $\mathrm{D}$. Detecting Chlamydia trachomatis by direct immunofluorescence using a Cytobrush sampling technique [letter; comment]. Genitourin Med 1989;65: 130.

3 Mårdh P-A, Zeeberg B. Toxic effect of sampling swabs and transportation test tubes on the formation of intracytoplasmic inclusions of Chlamydia trachomatis in McCoy cell cultures. Br $¥$ Veneral Dis 1981;57:268-72.

4 Lindner LE, Nettum JA, Miller SL, Altman KH. Comparison of scrape, swab, and cytobrush samples for the diagnosis of cervical chlamydial infection by immunofluorescence. Diagn Microbiol Infect Dis 1987; immunoflü

5 Ciotti RA, Sondheimer SJ, Nachamkin I. Detecting Chlamydia trachomatis by direct immunofluorescence using a Cytobrush sampling technique [published erratum appears in Genitourin Med 1989 Jan;65:62]. [see comments] . Genitourin Med 1988;64:245-6.

6 Svensson L, Bergelin I, Fryklund N, Ripa T. Effective sampling device and mucopurulent cervicitis in wome with chlamydial cervical infection. Acta Obstet Gynecol Scand 1989;68:79-82.

7 Moncada J, Schachter J, Shipp M, Bolan G, Wilber J. Cytobrush in collection of cervical specimens for detection of Chlamydia trachomatis $f$ Clin Microbiol 1989;27:1863-6.

8 Kellogg JA, Seiple JW, Klinedinst JL, Levisky JS. Comparison of cytobrushes with swabs for recovery of endocervical cells and for Chlamydiazyme detection of Chlamydia trachomatis. $f$ Clin Microbiol 1992;30: 2988-90.

9 Weiland TL, Noller KL, Smith TF, Ory SJ. Comparison of dacron-tipped applicator and cytobrush for detection of chlamydial infections. $f$ Clin Microbiol 1988 26:2437-8.

10 Kellogg JA, Seiple JW, Levisky JS. Efficacy of duplicate genital specimens and repeated testing for confirming positive results for chlamydiazyme detection of Chlamydia trachomatis antigen. I Clin Microbiol 1989; 27:1218-21.

11 Less MI, Newnan DM, Plackett M, Traynor PW, Forsyth JR, Garland SM. A comparison of cytobrush and cotton swab sampling for the detection of Chlamydia trachomaswab sampling for the detection of Chlamydia trach
tis by cell culture. Genitourin Med 1990;66:267-9.

12 Szarewski A, Pompey A, Bertrand J, Bradbeer C. Use of the Cytobrush for concurrent endocervical cytology and the Cytobrush for concurrent endocervical cytology
chlamydia sampling. Genitourin Med 1990;66:205-7.

13 Mandal D, Ray TK, Richmond SJ, Goorney BP, Haye KR. Comparison of cotton wool swab and cytobrush for detection of chlamydial infection in women attending a genitourinary medicine clinic. Int $\mathfrak{f} S T D$ Aids 1991;2:49-51

14 Dunlop EM, Goh BT, Darougar S, Woodland R, Tripleculture tests for diagnosis of chlamydial infection of the female genital tract. Sex Transm Dis 1985;12:68-71.

15 Munday PE, Carder JM, Hanna NF, Taylor-Robinson D. Is one swab enough to detect chlamydial infection of the cervix? Br₹ Venereal Dis 1984;60:384-6.

16 Svensson LO, Mares I, Olsson SE, Nordstrom MI Screening for Chlamydia trachomatis infection in women and aspects of the laboratory diagnostics. Acta Obstet Gynecol Scand 1991;70:587-90. 